\title{
Validación del Instrumento de Evaluación de la Conciencia Morfológica Oral (IECMO)
}

\author{
Lorena González-Sánchez ${ }^{1}$, Trinidad García², Débora Areces ${ }^{2}$, \\ Estrella Fernández ${ }^{2}$, Olga Arias-Gundin ${ }^{1}$ y Celestino Rodríguez ${ }^{2}$ \\ ${ }^{1}$ Universidad de León (España); ${ }^{2}$ Universidad de Oviedo (España)
}

\begin{abstract}
La conciencia morfológica puede jugar un papel relevante en lo que al proceso de alfabetización se refiere. Se presenta un estudio de validación experimental con una muestra de alumnos pre-lectores de Educación Infantil y alumnos de primero de Educación Primaria del Instrumento de Evaluación de la Conciencia Morfológica Oral (IECMO) diseñado para su estudio en español y centrada en la evaluación de las tres dimensiones de la conciencia morfológica: a) la morfología inflexional; b) la morfología derivacional y, c) las palabras compuestas. Participaron un total de cincuenta alumnos y alumnas pertenecientes al último curso de Educación Infantil y primero de Educación Primaria. Sus edades estaban comprendidas entre los 5 y los 7 años. En relación con el estudio experimental de validación se presentan los resultados iniciales de fiabilidad de la prueba por consistencia interna, análisis de ítems y estabilidad temporal análisis test-retest; y la validez de la prueba, tanto de contenido o aparente y de constructo o teórica. Los resultados obtenidos en el análisis de las propiedades psicométricas del instrumento permiten verificar tanto la validez, de contenido y de constructo, como la fiabilidad del IECMO. Este instrumento puede ser de gran utilidad, ya que el profesorado debería tener en cuenta el papel que juega la conciencia morfológica en el aprendizaje y dominio de diferentes habilidades metalingüísticas.
\end{abstract}

Palabras clave: Aprendizaje, conciencia morfológica, derivacional, lectura, morfología inflexional, palabras compuestas, evaluación.

Validation morphological oral awareness instrument. Morphological awareness can play an important role in the process of literacy refers. It presents an experimental validation study with a sample of students pre-readers in kindergarten and first students of Primary Education on Instrumento de Evaluación de la Conciencia Morfológica Oral (IECMO) designed for study Spanish and focuses on the evaluation of the three dimensions of morphological awareness: a) the morphology inflexional, b) derivacional morphology, and c) compound words. This study involved a total of fifty students belonging to the last year of kindergarten and first grade education. Their ages ranged from 5 to 7 years. In conjunction with experimental validation study presented the initial results of reliability testing for internal consistency, item analysis and stable test-retest analysis and the validity of the proof, or apparent conflict of content and construct or theoretical. The results obtained in the analysis of the psychometric properties of the instrument to verify verify both the validity of content and construct, as the reliability of IECMO. This tool can be very useful, since teachers should take into account the role of morphological awareness in learning and mastering different metalinguistic skills.

Keywords: Compound words, derivacional, evaluation, learning, morphological awareness, read, morphology inflexional.

Correspondencia: Celestino Rodríguez. Departamento de Psicología. Área de Psicología Evolutiva y de la Educación. Universidad de Oviedo. Plaza Feijoo s/n. C.P.: 33003. Oviedo (España). E-mail: rodriguezcelestino@uniovi.es 
La conciencia lingüística en el niño es un fenómeno en desarrollo que implica aptitudes cognitivas de forma combinada con aptitudes lingüísticas. Las posibles limitaciones tanto en las aptitudes cognitivas como lingüísticas condicionarían la emergencia de dicha conciencia. Hay que considerar que la conciencia lingüística se asocia con la adquisición exitosa de la lectura y la escritura (Seymour, 1997), la cual hace posible a los niños conocer los caminos que llevan del lenguaje oral al escrito, a la vez que proporciona las bases para adquirir de forma más profunda la comprensión de las formas y funciones del lenguaje oral.

La conciencia lingüística está compuesta de diversas áreas. Dentro de éstas, la capacidad de convertir operaciones mentales en unidades del habla es lo que se denomina conciencia fonológica (Morais, 1991), y es considerada como una de las más relevantes dentro de las que componen la conciencia lingüística, ya que los posibles déficits que puedan surgir en este área van a suponer problemas graves en la decodificación necesaria para la lectura y la escritura. Pero además de ésta, debemos tener en cuenta otras áreas consideradas como críticas en el desarrollo de las diferentes capacidades del lenguaje. Nos referimos a la conciencia gramatical y la conciencia morfológica (Moats, 2000). Al igual que la conciencia fonológica, la conciencia morfológica ayuda al proceso de decodificación de palabras escritas. Así, los morfemas cumplen funciones de bloque de construcción eficientes puesto que diferentes partes de las palabras (raíces o afijos base) se usan sistemáticamente para componer palabras nuevas y más largas, lo que permite reducir éstas en unidades más pequeñas (morfemas) que ayudan a la pronunciación, deletreo y significado. Cuando esta capacidad de análisis morfémico en el niño se lleva a la escritura con una mayor regularidad que en el habla, la conciencia morfémica en el conocimiento ortográfico conlleva al dominio de la escritura y, en consecuencia, del lenguaje oral y escrito (Carlisle, 2003).

La conciencia morfológica puede jugar un papel relevante en lo que al proceso de alfabetización se refiere, lo cual ha quedado reflejado en diferentes estudios llevados a cabo sobre la relación de este proceso con la escritura (García y González, 2006; Green et al., 2003) con la lectura (Cassalis, Colé, y Sopo, 2004; Clin, Wade-Woolley, y Heggie, 2009; Rodrigo et al., 2004) y con otra serie de procesos que pueden incidir de manera más o menos directa en alguna de las relaciones anteriores (Duncan, Cassalis, y Colé, 2009; McBride-Chang et al., 2005; Nunes y Bryant, 2004). De esta forma, podría decirse que el estudio de la conciencia morfológica se realiza en base a tres grandes áreas, entre las que se puede decir que dos de ellas priman en importancia a la hora de ser estudiadas y de desarrollar instrumentos o tareas de evaluación para tal fin. Estas dos grandes áreas son el lenguaje oral y la lectura, aunque se ha de admitir que desde hace unas décadas el estudio del papel que los diferentes procesos psicológicos desempeñan en la tarea de la composición escrita y en 
el aprendizaje de ésta ha sido tema prioritario para la gran mayoría de los investigadores, aunque no en lo que a conciencia morfológica se refiere.

En este sentido, los instrumentos diseñados para evaluar conciencia morfológica están determinados tanto por el área de estudio como el tipo de estudio que se desarrolla (descriptivo, instruccional o experimental). Y es que, de forma general, excepto en el caso de los estudios instruccionales, para los que se han diseñado programas acordes e inéditos sobre la conciencia morfológica, en lo que a evaluación se refiere, se hacen deseables la generalización de aquellos tests (que no uso de tareas independientes) que, en pocos casos, han sido diseñados expresamente para la evaluación de la conciencia morfológica, tanto en el campo de la lectura, como en el de la escritura. En consecuencia, las tareas empleadas en la evaluación dependerán del área concreta en la que queramos evaluar, si bien es cierto que no existen muchas diferencias cuando nos centramos en el vocabulario y la lectura, pero sí existen cuando evaluamos escritura. Cuando se trata de intervenir en conciencia morfológica se puede decir que los estudios llevados a cabo son bastante más escasos. De la misma forma, no existen suficientes programas destinados a la recuperación de las dificultades que puedan surgir en este aspecto del lenguaje, si bien es cierto que, en los pocos programas que se han podido consultar, no se aprecian diferencias significativas en función del área del lenguaje centrado en trabajar la conciencia morfológica (González, Rodríguez, Gázquez, González-Castro, y Álvarez, 2011).

Por todo ello, se hacen deseables la generalización de aquellos tests (que no uso de tareas independientes) que, en pocos casos, han sido diseñados expresamente para la evaluación de la conciencia morfológica, tanto en el campo de la lectura (Carlisle, 1995; McBride-Chang et al., 2005), como el de la escritura (García y González, 2006), así como estudios que indaguen en la conciencia morfológica ya sea desde un punto de vista descriptivo, instruccional o experimental (Arnbak y Elbro, 2000; Jiménez y del Cristo, 2017; Nunes, Bryant, y Olson, 2003). Así, el objetivo del presente trabajo es mostrar un instrumento diseñado para evaluar conciencia morfológica en función de dos planos: el plano de producción morfológica y el plano de juicio morfológico.

\section{MÉTODO}

\section{Participantes}

En el presente estudio participaron un total de cincuenta alumnos y alumnas pertenecientes al último curso de Educación Infantil y primero de Educación Primaria, si bien es cierto que, al comienzo del mismo, es decir, en el primer seguimiento realizado, los alumnos eran sesenta y nueve. Sus edades estaban comprendidas entre los 5 y los 7 años, quedando distribuidos en función del género y curso tal y como aparecen en la 
tabla 1. Ninguno de los participantes presentaba necesidades educativas especiales, ya que su nivel de competencia curricular se encontraba dentro de las medias correspondientes a cada uno de los cursos de infantil y primaria con los que se trabajó.

Finalmente, los alumnos provenían de tres colegios diferentes siendo éstos representativos de la capital y provincia de León.

Tabla 1. Distribución de la muestra por curso, género y edad

\begin{tabular}{lccccc}
\hline & & Seguimiento $1^{\circ}$ & Seguimiento $2^{\circ}$ & Seguimiento $3^{\circ}$ & Total \\
\cline { 2 - 6 } & Curso & $3^{\circ} \mathrm{EI}$ & $1^{\circ} \mathrm{EP}($ Comienzo curso) & $1^{\circ} \mathrm{EP}$ (final curso) & $3^{\circ} \mathrm{EI}-1^{\circ} \mathrm{EP}$ \\
\hline \multirow{2}{*}{ Género } & Niños & 35 & 27 & 27 & 27 \\
\cline { 2 - 6 } & Niñas & 34 & 25 & 23 & 23 \\
\hline Total curso & 69 & 52 & 60 & 50 \\
\hline \multicolumn{2}{l}{ Media edad curso } & 5.6 & 6.2 & 6.7 & 6.1 \\
\hline Edad mín-max & $5.1-6.5$ & $5.7-7.0$ & $6.4-7.3$ & $5.1-7$ \\
\hline
\end{tabular}

\section{Instrumento}

El Instrumento de Evaluación de la Conciencia Morfológica Oral (IECMO) es una adaptación del Instrumento de Evaluación de la Conciencia Morfológica en Escritura (IECME) (García y González, 2006). Se trata de un instrumento específico dirigido a alumnos de último curso de Educación Infantil a $2^{\circ}$ de Educación Primaria, cuyo fin es evaluar conciencia morfológica en función del plano de producción morfológica y del plano de juicio morfológico. Ambos planos, a su vez, están divididos en tareas que estiman diferentes aspectos de la conciencia morfológica (ver tabla 2).

Tabla 2. Aspectos evaluados para cada una de las tareas y planos del IECMO

\begin{tabular}{ll}
\hline \multicolumn{1}{c}{ Instrumento de Evaluación de la Conciencia Morfológica Oral } \\
\hline \multicolumn{1}{c}{ Plano Producción morfológica } & \multicolumn{1}{c}{ Plano Juicio morfológico } \\
\hline 1 Género & Identificar \\
\hline 2 Género & 2 Género \\
\hline 3 Número & 3 Número \\
\hline 4 Número & 4 Número \\
\hline 5 Verbos & 5 Verbos \\
\hline 6 Verbos & 6 Verbos \\
\hline 7 Prefijos & 7 Prefijos \\
\hline 8 Prefijos & 8 Prefijos \\
\hline 9 Sufijos & 9 Sufijos \\
\hline 10 Sufijos & 10 Sufijos \\
\hline 11 Palabras derivadas & 11 Palabras derivadas \\
\hline 12 Palabras derivadas & 12 Palabras derivadas \\
\hline 13 Palabras Compuestas & 13 Familia de palabras \\
\hline 14 Familia de palabras & 14 Familia de palabras \\
\hline 15 Familia de palabras & 15 Palabras Compuestas \\
\hline
\end{tabular}

El plano de juicio morfológico mide lenguaje receptivo en función de la capacidad del alumno a la hora de juzgar y establecer posibles relaciones morfológicas entre las palabras. Se tiene así en cuenta el establecimiento de juicios con palabras formadas por inflexiones, con palabras derivadas de relaciones transparentes y aquellas 
que implican cambios fonológicos. En dicho plano se evalúa la tarea de "Identificar". En el caso de los ejemplares que componen "Identificar", el alumno deberá juzgar la relación morfológica correcta de entre diferentes posibilidades que se le ofrecen. En este tipo de ejemplares el alumno va a contar siempre con una palabra dada sobre la que tendrá que realizar el análisis morfológico que se le requiera (ver figura 3). "Identificar" está compuesta por quince ejemplares de tarea, dos ejemplares que evalúan el género, dos que evalúan el número, dos de verbos, dos de prefijos, dos de sufijos, dos de palabras primitivas y derivadas, dos de familias de palabras, y uno de palabras simples y compuestas. Finalmente, la máxima puntuación a obtener en ella es de 30 puntos, ya que cada uno de los ejemplares que la componen se evalúa de 0 a 2 puntos en función de su grado de corrección. Además, se ha creído conveniente obtener una puntuación referente a errores, la cual puede ser de 36 puntos.

Figura 1. Ejemplos de tarea "Identificar" para evaluar Juicio Morfológico

4) Presta atención: dos balons. Está mal porque si decimos dos no decimos balons, sino

balones que si está bien dicho. Fíjate en estas palabras y di cuáles están mal si decimos que bay dos.
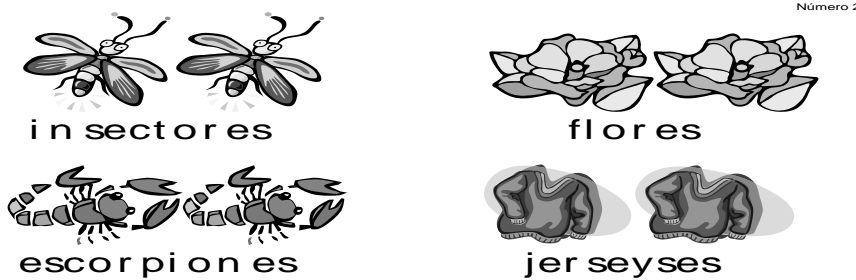

Con el plano de la producción morfológica se evalúa el lenguaje expresivo, produciendo las formas correctas de las palabras requeridas que, en su mayoría, están formadas por inflexiones, aunque también se presentan palabras derivadas de relaciones transparentes y otras que implican cambios fonológicos. Los ejemplares de tareas que componen "Completar" pretenden evaluar conciencia morfológica produciendo la forma correcta de palabras, bien sea de palabras que no están completas, series sin finalizar, etc., (ver figura 4). La presente tarea está compuesta por un total de quince ejemplares. Cada uno de ellos precisa de una explicación específica, si bien es cierto que hay algunas normas que son de tipo general y que conviene que se tengan presentes antes de comenzar la prueba. En concreto, la tarea de completar está constituida por dos ejemplares que evalúan el género, dos que evalúan el número, dos de verbos, dos de prefijos, dos de sufijos, dos de palabras primitivas y derivadas, dos de familias de palabras, y uno de palabras simples y compuestas. De nuevo, la máxima puntuación a obtener en ella es de 30 puntos, ya que cada uno de los ejemplares que la componen se 
evalúan de 0 a 2 puntos en función de su grado de corrección. Además, se obtiene, nuevamente, una puntuación referente a errores, la cual puede ser en este caso de 30 puntos. La administración del IECMO es oral e individual.

Finalmente, con el IECMO se obtiene una doble puntuación. Por un lado, se consigue el número total de aciertos conseguidos por el niño tanto por planos del instrumento como del total, pero, por otro lado, se obtiene una puntuación sobre los errores efectuados, de nuevo tanto en lo que se refiere a cada uno de los planos como de manera total. Se decidió incluir una puntuación de errores ya que se consideró relevante el conocer el tipo de ítems con los que los niños podían tener más dificultades, constituyendo éstos una fuente de información valiosa a la hora de determinar el tipo de estrategia que utiliza cada alumno.

\begin{tabular}{l} 
Figura 2. Ejemplo de tarea "Completa" para evaluar Producción Morfológica \\
8) Dime cómo se llaman los siguientes señores que trabajan con las siguientes cosas o en los siguientes lugares completando las palabra \\
que te damos: \\
El señor que bace pan es un... \\
\hline
\end{tabular}

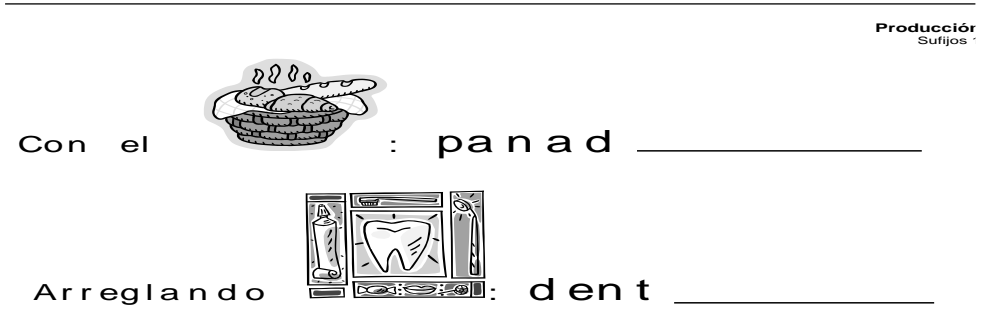

\section{Procedimiento}

Se comenzó por realizar la oportuna revisión bibliográfica con el fin de fundamentar teóricamente la selección de las diferentes tareas y ejemplares de tareas que componen el IECMO, otorgándole así validez de contenido.

Este estudio se encuadra dentro de una investigación más amplia sobre la influencia de la conciencia morfológica en el aprendizaje de la lectura y la escritura. Dicha investigación se estructuraba en tres momentos de evaluación, tres momentos en los que también se administró el IECMO. Siempre se siguió la misma estructura: de forma individual, en horario académico, en sesiones de unos 45 minutos y en un aula especial acondicionada para tal fin. Los niños realizaban los ejemplares de tareas pertenecientes a los dos tipos de tareas en las que se distribuyen los planos de producción morfológica y juicio morfológico. Así, para el juicio morfológico realizaban quince ejemplares pertenecientes a la tarea de "Identificar" y para la producción morfológica, se contaban con otros quince ejemplares pertenecientes a las tareas de "Completar". El evaluador debía asegurarse de que el niño se encontraba lo 
suficientemente motivado para la aplicación de la prueba, interrumpiendo la misma si se observaba cansancio por parte del mismo. Además, era conveniente que el aplicador se situase cerca del niño, para que, en los momentos en los que se estuvieran dando instrucciones, éste señalase y el alumno observara sin problemas. Cada uno de los ejemplares de tareas comenzaba con un ítem ejemplo que estaba separado del ejemplar en sí en una hoja aparte. El aplicador debía asegurarse de que el niño había entendido bien la tarea antes de comenzar con el resto de los ítems. Finalmente, el evaluador contaba con una hoja de registro en la que debía anotar las respuestas dadas por el niño en función de lo requerido para cada ejemplar.

Una vez finalizada la evaluación, se procedió a la corrección de los datos, los cuales fueron codificados e informatizados adecuadamente para su posterior análisis estadístico.

\section{Análisis de datos}

Una vez finalizado el proceso de corrección, codificación e informatización de datos, se realizaron diferentes análisis estadísticos mediante el paquete informático SPSS 17.0. Se realizaron todos aquellos análisis relacionados con las propiedades psicométricas del IECMO, así como de cada una de las tareas y ejemplares que lo componen. Se proporcionó así la información necesaria para obtener datos concluyentes acerca de la fiabilidad y la validez que es necesaria para evaluar diferentes aspectos implicados en la conciencia morfológica. Para la primera de ellas, se analiza la consistencia interna, considerando tanto sus elementos como tareas, la calidad del instrumento a través del análisis de los ítems, así como la consistencia del mismo a través del análisis test-retest. En cuanto a la validez, se ha tenido en cuenta tanto la validez de contenido o aparente, como la de constructo o teórica, y la validez estructural y predictiva.

\section{RESULTADOS}

\section{Fiabilidad}

Para determinar la fiabilidad del IECMO se realizaron tres tipos de análisis diferentes. Para comenzar, se realizó el análisis de la calidad de los ítems que componen el instrumento calculando los estadísticos descriptivos de cada uno de ellos; a continuación se llevó a cabo el análisis de fiabilidad por consistencia interna, para la que se utilizó el $\alpha$ de Cronbach; en tercer lugar, y con el fin de comprobar su consistencia en diferentes momentos de aplicación, se procedió a realizar un análisis test-retest. 


\section{Análisis de los ítems}

En la tabla 5 pueden observarse los estadísticos de los ítems relativos a los totales en aciertos que componen el IECMO para cada uno de los tres momentos de aplicación. El análisis descriptivo de los resultados nos permite revelar que en lo referente a la puntuación general del total de aciertos sobre conciencia morfológica, las

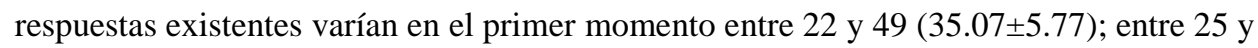
$53(38.79 \pm 6.15)$ en el segundo de los momentos; y entre 0 y $53(42.17 \pm 9.93)$ en el tercer y último momento. Los resultados de la asimetría y curtosis de nuevo para la puntuación general del total de aciertos sobre conciencia morfológica para el primer y segundo momento (.074 y -.516 para el primer momento y .039 y -.365 para el segundo), permiten concluir que el instrumento en ambas medidas muestra una notable sensibilidad. No ocurre lo mismo en el tercero de los momentos de aplicación, ya que la asimetría de esa misma variable es de -3.32 y la curtosis de 12.60 .

Tabla 5. Estadísticos descriptivos de las puntuaciones totales del IECMO en los tres momentos de aplicación

\begin{tabular}{lccccc}
\hline \multicolumn{7}{c}{ Variable } & Media & Desv. Típica & Varianza & Asimetría & Curtosis \\
\hline \multicolumn{7}{c}{$1^{\mathrm{er}}$ Momento } & & & -.17 \\
\hline Juicio Identificar Total Aciertos & 19.80 & 2.97 & 8.84 & -.08 & -.60 \\
\hline Producción Completar Total Aciertos & 15.28 & 4.15 & 17.23 & .05 & -.51 \\
\hline Total Conciencia Morfológica Aciertos & 35.07 & 5.77 & 33.30 & .07 & \\
\hline \multicolumn{7}{c}{$2^{\circ}$ Momento } \\
\hline Juicio Identificar Total Aciertos & 21.08 & 2.86 & 7.87 & .09 & -.67 \\
\hline Producción Completar Total Aciertos & 17.71 & 4.19 & 17.58 & -.01 & -.23 \\
\hline Total Conciencia Morfológica Aciertos & 38.79 & 6.15 & 37.85 & .03 & -.36 \\
\hline \multicolumn{7}{c}{$3^{\text {er Momento }}$} & & & \\
\hline Juicio Identificar Total Aciertos & 21.04 & 4.85 & 23.52 & -2.19 & 6.33 \\
\hline Producción Completar Total Aciertos & 21.13 & 5.59 & 31.25 & -3.01 & 11.38 \\
\hline Total Conciencia Morfológica Aciertos & 42.17 & 9.93 & 98.61 & -3.32 & 12.60 \\
\hline
\end{tabular}

Nota. Para el primero de los momentos $\mathrm{N}=69$, mientras que para el segundo y tercer momento $N=52$.

\section{Consistencia interna: $\alpha$ de Cronbach}

A través de la utilización del $\alpha$ de Cronbach para hallar el índice de fiabilidad del instrumento, podemos deducir que éste, al presentarse de forma completa, atendiendo a la puntuación aciertos como a la puntuación errores en el último de los seguimientos realizados, posee una alta fiabilidad ya que presenta un $\alpha$ de Cronbach de 0.854 . Si por el contrario nos detenemos a analizar el IECMO en función de sus planos, observamos que tanto la producción morfológica aciertos ( $\alpha$ de Cronbach=.836) y producción morfológica errores ( $\alpha$ de Cronbach=.850) como el juicio morfológico aciertos ( $\alpha$ de Cronbach=0.828) y juicio morfológico aciertos ( $\alpha$ de Cronbach=.843) muestran así mismo una alta fiabilidad. Por otro lado, y si atendemos a los resultados obtenidos en los tres momentos de evaluación y centrándonos únicamente en la puntuación obtenida sobre los aciertos, la fiabilidad parece confirmase de nuevo, ya que el instrumento posee un $\alpha$ de Cronbach de 0.912. Finalmente, la consistencia interna del instrumento parece 
asegurarse si observamos la presentación completa del mismo, producción y juicio morfológico, en relación a las puntuaciones de aciertos y errores de los tres momentos en los que se tomaron medidas, ya que el IECMO muestra un grado de fiabilidad aceptable con un $\alpha$ de Cronbach de .786. En la tabla 6 se recogen los resultados respecto a la fiabilidad de los totales tanto de aciertos como de errores en conciencia morfológica para cada uno de los tres momentos en los que se tomaron medidas.

Tabla 6. Resultados de fiabilidad de los totales del IECMO en los tres momentos de evaluación

\begin{tabular}{lcccc}
\hline \multicolumn{1}{c}{ Variable } & $N$ & Media & Desviación típica & $\alpha$ de Cronbach \\
\hline Total Conciencia Mofológica Aciertos & 52 & 35.38 & 5.85 & .780 \\
\hline Total Conciencia Mofológica Errores & 52 & 22.15 & 6.02 & .806 \\
\hline Total Conciencia Mofológica Aciertos 2 & 52 & 38.79 & 6.15 & .770 \\
\hline Total Conciencia Mofológica Errores 2 & 52 & 18.37 & 5.20 & .775 \\
\hline Total Conciencia Mofológica Aciertos 3 & 52 & 42.17 & 9.93 & .759 \\
\hline Total Conciencia Mofológica Errores 3 & 52 & 14.90 & 5.42 & .775 \\
\hline
\end{tabular}

Si se consideran las $\alpha$ de Cronbach de cada elemento, y se analiza la posibilidad de su eliminación, la consistencia interna del instrumento apenas varía, lo que indica que la contribución de cada uno de ellos en la fiabilidad del instrumento es similar, por lo que no hay ninguna contribución especial de ninguno, y por lo tanto, su inclusión conjunta parece la mejor opción.

\section{Estabilidad temporal: análisis test-retest}

En la tabla 7 se presentan las correlaciones test-retest de las puntuaciones relativas al Total Conciencia Morfológica Aciertos, y Total Conciencia Morfológica Errores. Se realizaron correlaciones test-retest para comparar cada uno de los tres momentos de aplicación entre sí. Este análisis proporciona una medida de estabilidad temporal de las puntuaciones obtenidas al aplicar el mismo test en distintas ocasiones. De esta forma, la correlación del Total Conciencia Morfológica Aciertos del primer momento de medida con el segundo es de .72 y de .40 cuando correlaciona con el tercero de los momentos. Cuando se compara el segundo momento con el tercero, la correlación entre las puntuaciones del Total Conciencia Morfológica Aciertos es de .73. En el caso de Total Conciencia Morfológica Errores, las correlaciones entre la primera medida y la segunda es de .49, mientras que la correlación entre el primer momento y el tercero es de .21. Si se observa la correlación existente entre las medidas de Total Conciencia Morfológica errores entre el segundo y tercer momento de medida, ésta es de 32 .

En síntesis, los datos indican la existencia de una alta estabilidad de las medidas en los tres momentos distintos de aplicación. 
GONZÁLEZ-SÁNCHEZ et al. Instrumento evaluación Conciencia morfológica oral

Tabla 7. Correlación entre medidas principales del IECMO en tres momentos de aplicación

\begin{tabular}{lccccccc}
\hline \multirow{2}{*}{ Variable } & \multicolumn{7}{c}{ Medida } \\
\cline { 2 - 8 } & \multicolumn{2}{c}{$1^{\text {er }}-2^{\text {e }}$ momento } & \multicolumn{1}{c}{$1^{\text {er }}-3^{\text {er }}$ momento } & $2^{\text {o }}-3^{\text {er }}$ momento \\
\cline { 2 - 7 } & $r$ de Pearson & $p$ & $r$ de Pearson & $p$ & $r$ de Pearson & $p$ \\
\hline Total Conciencia Morfológica Aciertos & .723 & .001 & .405 & .003 & .734 & .001 \\
\hline Total Conciencia Morfológica Errores & .495 & .001 & .213 & .129 & .323 & .020 \\
\hline
\end{tabular}

\section{Validez}

Para analizar la validez del Instrumento de Evaluación de la Conciencia Morfológica Oral, se efectuaron análisis de la validez de contenido o aparente, análisis de validez de constructo o teórica y de la validez predictiva del mismo.

\section{Validez de contenido}

La validez de contenido del IECMO parece apropiada ya que su diseño y elaboración se ha basado en los aspectos que diferentes estudios analizados, tanto teóricos como empíricos, consideran acerca del concepto de conciencia morfológica y, al igual que había ocurrido con el IECME, ya que se trata de una adaptación de éste, a partir de lo que la Real Academia Española de la Lengua considera como elementos que forman parte de la morfología española, desarrollado en el capítulo dos de la presente tesis doctoral. En concreto, se recogen los contenidos morfológicos relativos a género, número, verbos, prefijos, sufijos, palabras derivadas, familias de palabras y palabras compuestas, y ello tanto desde la producción morfológica como desde el juicio morfológico. Para el caso de la producción morfológica, también se presentan ejemplares pertenecientes a la tarea completar, a través de la cual se evalúa el lenguaje productivo. E, igualmente para juicio morfológico, se presentan ejemplares de la tarea identificar, con ejemplares de cada uno de los contenidos de morfología del español y que miden el lenguaje receptivo.

\section{Validez de constructo}

La validez de constructo se halló mediante un análisis factorial de componentes principales por rotación varimax normalizada a partir de todas las puntuaciones totales directas de los parámetros evaluados por el instrumento en el primer momento de evaluación. Según el análisis factorial del IECMO se encuentra que los seis primeros factores explican el $68.78 \%$ de la varianza total acumulada, tal como se muestra en la tabla 8 . El primero de los factores obtenidos, que explica el $28.72 \%$ de la varianza total, corresponde al factor que podríamos denominar producción morfológica, dado que en general, está saturado por los pesos factoriales de las puntuaciones totales obtenidas en relación a este plano de evaluación concreto. El segundo factor, el cual podríamos denominar juicio morfológico y que consecuentemente está saturado por medidas de ese plano, explica el $12.08 \%$ de la varianza total. En concreto, se refiere a la tarea de identificar, y a los ejemplares de tarea de número, sufijos, familia de palabras y 
puntuación total en juicio morfológico, todo ello en lo que al total de aciertos se refiere. El tercer factor, de nuevo está saturado por las medidas de juicio morfológico2, en concreto, por los ejemplares de la tarea de identificar en lo referente a prefijos, palabras derivadas, de nuevo familia de palabras y puntuación total en juicio morfológico, explicando el $9.11 \%$ de la varianza total. El cuarto factor de juicio morfológico y producción morfológica, se refiere exactamente a las tareas identificar y completar respectivamente, explica el $6.60 \%$ de la varianza total, y está saturado por los ítems correspondientes género y número para el primero, y número para el segundo. El quinto factor, al cual se puede denominar juicio morfológico y producción morfológica verbos, se centra en la tarea de completar e identificar saturadas por las medidas relativas a verbos, explicando el $6.36 \%$ de la varianza total. El sexto y último factor propuesto, producción morfológica 2 se refiere a los contenidos de la tarea de completar, explica el $5.88 \%$ de la varianza total, y está saturado en este caso por las medidas de los contenidos de género y de familia de palabras.

Tabla 8. Varianza explicada por cada uno de los factores obtenidos mediante un análisis factorial de componentes principales con rotación varimax normalizada

\begin{tabular}{lcc}
\hline \multicolumn{1}{c}{ Componente o factor } & \% varianza & $\%$ acumulado \\
\hline 1. Producción morfológica & 28.727 & 28.727 \\
\hline 2. Juicio morfológico & 12.088 & 40.814 \\
\hline 3. Juicio morfológico2 & 9.116 & 49.931 \\
\hline 4. Juicio morfológico y producción morfológica & 6.603 & 56.534 \\
\hline 5. Juicio morfológico y producción morfológica verbos & 6.386 & 62.897 \\
\hline 6. Producción morfológica2 & 5.888 & 68.785 \\
\hline
\end{tabular}

La denominación de los factores resultantes se ha establecido en función del análisis de componentes principales según el método de rotación normalizado, varimax con Kaiser, que se muestra en la tabla 9 y en la que se recogen para cada factor los parámetros que lo saturan. De esta forma, y como ha sido expuesto anteriormente, los tres primeros factores explican casi el $50 \%$ de la varianza, en concreto, el $49.93 \%$. El primero de ellos explica el $28.72 \%$ de la varianza total, y está saturado por los pesos factoriales de siete medidas correspondientes a producción morfológica, que hacen referencia a la tarea de completar, principalmente al ejemplar referente a sufijos aciertos con un peso factorial de .663, al ejemplar palabras derivadas aciertos con un peso factorial de .717 y al ejemplar referido al total de aciertos obtenidos en producción morfológica, con un peso de .918 .

En lo referente al segundo factor, el cual explica el $12.08 \%$ de la varianza total, y el cual corresponde a juicio morfológico, está saturado por un total de cuatro variables. De todas ellas, especialmente significativas son las variables de juicio identificar sufijos total aciertos, la cual posee un peso factorial de .803 , y juicio identificar total aciertos, con un peso de .551. La suma de cuatro ejemplares de juicio morfológico 2 supone el tercero de los seis factores, explica el $9.11 \%$ de la varianza total 
y está saturado por cuatro medidas: juicio identificar prefijos total aciertos, con un peso factorial de .676, juicio identificar palabras derivadas total aciertos con .642 de peso factorial, juicio identificar familia de palabras total aciertos cuyo peso es de .508, y juicio identificar total aciertos con .694 de peso factorial. El cuarto factor está saturado por tres medidas de juicio morfológico y producción morfológica y explican el $6.60 \%$ de la varianza total. En concreto, las variables que saturan el factor son juicio identificar género total aciertos, que tiene un peso factorial de .741, juicio identificar número total aciertos, cuyo peso es de .506 , y producción completar número total aciertos, con un peso de .600. El quinto factor de nuevo hace referencia a juicio morfológico y producción morfológica, explicando el $6.36 \%$ de la varianza, pero en esta ocasión en lo que se refiere a verbos, estando saturado por las medidas de juicio identificar verbos total aciertos, cuyo pero factorial es de .722, y de producción completar verbos total aciertos, con un peso de .535. Finalmente, el sexto factor, producción morfológica 2 que explica el $5.88 \%$ de la varianza total acumulada, es saturado por dos parámetros, producción completar género aciertos, que tiene un peso factorial de .874 y producción completar familia de palabras cuyo peso factorial es de .460 .

Tabla 9. Factores obtenidos mediante análisis factorial de componentes principales con rotación varimax normalizada y parámetros que los saturan

\begin{tabular}{ll}
\hline \multicolumn{2}{c}{ Factor 1: Producción morfológica } \\
\hline \multicolumn{2}{c}{ Factores o componentes y variables } \\
\hline Producción Completar Número: total aciertos & .478 \\
\hline Producción Completar Sufijos: total aciertos & .663 \\
\hline Producción Completar Prefijos: total aciertos & .473 \\
\hline Producción Completar Palabras Derivadas: total aciertos & .717 \\
\hline Producción Completar Verbos: total aciertos & .484 \\
\hline Producción Completar Familia Palabras: total aciertos & .644 \\
\hline Producción Completar Total Aciertos & .918 \\
\hline \multicolumn{2}{c}{ Fotal Conciencia Morfológica 2: Juicio Morfológico } \\
\hline \multicolumn{2}{c}{ Factor 3: Juicio Morfológico2 } \\
\hline Juicio Identificar Número: total aciertos \\
\hline Juicio Identificar Sufijos: total aciertos \\
\hline Juicio Identificar Familia Palabras: total aciertos \\
\hline Juicio Identificar Total Aciertos \\
\hline \multicolumn{2}{c}{.733} \\
\hline Juicio Identificar Prefijos: total aciertos & .519 \\
\hline Juicio Identificar Palabras Derivadas: total aciertos & .803 \\
\hline Juicio Identificar Familia Palabras: total aciertos & .479 \\
\hline Juicio Identificar Total Aciertos & .676 \\
\hline \multicolumn{2}{c}{ Factor 4: Juicio Morfológico y Producción Morfológica- } \\
\hline Juicio Identificar Género: total aciertos & .694 \\
\hline Juicio Identificar Número: total aciertos & .741 \\
\hline Producción Completar Número: total aciertos & .506 \\
\hline \multicolumn{2}{c}{ Factor 5: Juicio Morfológico y Producción Morfológica Verbos } \\
\hline Juicio Identificar Verbos: total aciertos & .675 \\
\hline Producción Completar Verbos: total aciertos & .535 \\
\hline \multicolumn{2}{c}{ Factor 6: Producción Morfolóigica2 } \\
\hline Producción Completar Género: total aciertos \\
\hline Producción Completar Familia Palabras: total aciertos \\
\hline
\end{tabular}




\section{Validez predictiva}

La validez predictiva queda justificada por el estudio de predicción que se presenta a continuación. El hecho de que se obtenga una estructura concreta de predicciones se relaciona con el modelo teórico que hemos de postular y que se viene desprendiendo de la revisión teórica realizada y que los datos aquí presentados y evidencias matizan o confirman, según las explicaciones que siguen.

\section{Validez estructural}

El hecho de que se utilicen las medidas de conciencia morfológica en tres momentos diferentes da pie a conocer la estructura de las relaciones entre sí y, en parte, aporte información relevante sobre la validez estructural, aunque ésta queda limitada por el uso de un único instrumento de conciencia morfológica, ya que el utilizado en el primer estudio no era aplicable a niños tan pequeños. El hecho de que no se dispongan, en español, de otras pruebas con las cuales comparar, limita este tipo de análisis, que hubiera sido deseable, aunque de forma indirecta. Los datos ofrecidos por otros estudios confirman la relación existente y la analogía y comparabilidad entre nuestros instrumentos y éstos desarrollados en otros estudios.

\section{DISCUSIÓN Y CONCLUSIONES}

Partiendo de los análisis realizados y de los diferentes resultados obtenidos a partir de los mismos, son varias las conclusiones que pueden hacerse acerca del presente estudio. Para comenzar, podría decirse que se verifica tanto la validez, de contenido y de constructo, como la fiabilidad del IECMO. Nos encontramos así con un instrumento que puede considerarse, en su conjunto, como un medio fiable para evaluar la doble dimensión en la que es posible estructurar la conciencia morfológica, esto es, juicio morfológico y producción morfológica, tal y como muestran el análisis de ítems, en el que se observa que el instrumento en ambas medidas muestra una notable sensibilidad, en el análisis de fiabilidad por consistencia interna, y en el análisis test-retest, a través del cual se observa una alta estabilidad de las medidas en los tres momentos distintos de aplicación.

En segundo lugar, los análisis factoriales realizados permiten extraer varias conclusiones. Así, el análisis factorial conjunto de todas las medidas totales del IECMO, permite confirmar la diferenciación de dos planos básicos en conciencia morfológica. El plano de producción morfológica está reflejado en el primer factor o componente de la prueba, mientras que el plano de juicio morfológico constituye el segundo de los factores extraídos. Se concluye así que la conciencia morfológica es mejor evaluada con tareas que correspondan a ambos planos. 
Por otra parte, los factores 3, 4 y 6 vienen a corroborar y matizar este hecho, mientras que el factor 5 se identifica con el parámetro de los verbos para cada uno de los dos planos.

Se confirma así la necesidad de evaluar la conciencia morfológica a través de dos planos (Carlisle, 1995). Y es que, según diversos estudios, tanto las tareas de producción como de recepción deberían ser usadas para evaluar dicho tipo de conciencia y, siguiendo las aportaciones de Rubin (1988), considerar así tanto el conocimiento explícito, medido a través de la capacidad para usar reglas morfológicas a la hora de crear nuevas palabras, como el conocimiento implícito, evaluado por la habilidad a la hora de identificar los diferentes morfemas que forman una palabra morfológicamente compleja.

Aún así, y partiendo de los buenos resultados obtenidos, es de aceptar que son necesarias ciertas modificaciones para la construcción definitiva del IECMO. Según los resultados, existen ejemplares de tareas que abordan los diferentes tipos de contenido del instrumento que resultan más fáciles que otras y que podrían resultar eliminados del instrumento. Este es el caso de aquellos que evalúan género, ya que, en los tres momentos de aplicación se obtuvo la mayor puntuación posible por la mayoría de los niños evaluados. Sin embargo, la validez de contenido realizada hace que se considere imprescindible la inclusión del mismo, dada la importancia de constatar la adquisición del género como otro elemento más de morfología.

Otra de las limitaciones encontradas se refiere al uso de las tareas seleccionadas. Éstas se ajustan a la faceta receptiva y expresiva del lenguaje, y tienen en cuenta tanto la parte implícita como explícita de la conciencia, pero se hubiera contribuido más a las conclusiones finales si se hubiera incluido una distinción entre aquellas tareas que evalúan morfología derivacional y morfología inflexional, al uso de lo que se ha venido haciendo en otros estudios (Cassalis y Alexandre, 2000; Deacon y Bryant, 2006; Mahony et al., 2000), lo cual mejoraría el conocimiento de la naturaleza de la conciencia morfológica.

Varias son las aportaciones que pueden derivarse de la validación de este instrumento. En cuanto al nivel teórico, podemos decir que, el partir de una prueba específicamente elaborada para evaluar conciencia morfológica, y en español, supone el inicio de futuros estudios encaminados a analizar aspectos como los que se investigan en el presente estudio pero dando un paso más allá, y comparando dichos resultados de niños sin dificultades de aprendizaje, con los resultados que pudieran obtener niños con dificultades de aprendizaje o con diferentes trastornos, como podría ser el caso de niños con deficiencia auditiva.

En cuanto al nivel práctico, el IECMO puede ser de gran utilidad, ya que el profesorado debería tener en cuenta el papel que juega la conciencia morfológica en el aprendizaje y dominio de diferentes habilidades metalingüísticas, como es el caso de la 
lectura (Aguilar, Aragón, Navarro, Delgado, y Marchena, 2017), y en menor proporción la escritura, contando para ello con la ayuda de un instrumento objetivo que les permita conocer el nivel de dicha conciencia en sus alumnos y así intervenir en consecuencia, y más aún tomando en consideración la puntuación errores que se genera y que puede orientar hacia el aspecto concreto en el que falla el niño. Se trataría así de un instrumento de fácil aplicación y corrección que les permitiría reorientar o adaptar su proceso de enseñanza.

\section{REFERENCIAS}

Aguilar, M., Aragón, E., Navarro, J.I., Delgado, C., y Marchena, E. (2017). Análisis del efecto san Mateo en un studio longitudinal sobre el desarrollo lector durante la educación primaria $\left(1^{\circ}\right.$ a $5^{\circ}$ ). European Journal of Education and Psychology 10, 23-32.

Arnbak, E., y Elbro, C. (2000). The effects of morphological awareness training on the reading and spelling skills of young dyslexics. Scandinavian Journal of Educational Research, 44(3), 229-251.

Carlisle, J.F. (1995). Morphological awareness and early ready achievement. En L.B. Feldman (Ed.), Morphological aspects of language processing (pp.189-209). Hillsdale, NJ: Erlbaum.

Carlisle, J.F. (2003). Morphology matters in learning to read: A commentary. Reading Psychology, 24, 291-322.

Cassalis, S., Colé, P., y Sopo, D. (2004). Morphological Awareness in Developmental Dyslexia. Annals of Dislexia, 54(1), 114-138.

Cassalis, S., y Louis-Alexandre, M.F. (2000). Morphological analysis, phonological analysis and learning to read French: a longitudinal study. Reading and Writing: An Interdisciplinary Journal, 12, 303-335.

Clin, E., Wade-Woolley, L., y Heggie, L. (2009). Prosodic sensitivity and morphological awareness in children reading. Journal of Experimental Child Psychology, 104, 197-213.

Deacon, H., y Bryant, P. (2006). This turnip's not for turning: Children's use of root morphemes in spelling. British Journal of Developmental Psychology, 24, 567-575.

Duncan, L., Cassalis, S., y Colé, P. (2009). Early metalinguistic awareness of derivational morphology: Observations from a comparison of English and French. Applied Psycholinguistics, 30, 405-440.

García, J.N., y González, L. (2006). Diferencias en la conciencia morfológica, la escritura y el lenguaje en función del desarrollo y el nivel educativo del niño. Psicothema, 18(2), 171179.

González, L., Rodríguez, C., Gázquez, J.J., González-Castro, P., y Álvarez, D. (2011). La conciencia morfológica: tendencias de desarrollo y patrón evolutivo en Educación Infantil y Primaria. Psicothema. 23(2), 239-244.

Green, L., McCutchen, D., Shwiebert, C., Quinlan, T., Eva-Wood, A., y Juelis, J. (2003). Morphological Development in Children's Writing. Journal of Educational Psychology, 95(4), 752-761. 
Jiménez, J.E., y del Cristo, S. (2017). Análisis factorial confirmatorio del Indicadores de Progreso de Aprendizaje en Matemáticas (IPAM) en escalas de primer curso de Primaria. European Journal of Invesstigation in Health, Psychology and Education, 7(1), 31-45.

Mahony, D., Singson, M., y Mann, V. (2000). Reading ability and sensitivity to morphological relations. Reading and Writing: An Interdisciplinary Journal, 12, 191-218.

McBride-Chang, C., Cho, J., Liu, H., Wagner, R., Shu, H., Zhou, A., Cheuk, C., y Muse, A. (2005). Changing models across cultures: associations of phonological awareness and morphological structure awareness with vocabulary and word recognition in second graders from Beijing, Hong Kong, Korea, and the United States. Journal of Experimental Child Psychology, 92, 140-160.

Moats, L. (2000). Speech to print: Language essentials for teachers. Baltimore: Brookes.

Morais, J. (1991). Constraints on the development of phonemic awareness. En S. Brady y D. Shankweiler (Eds.), Phonological processes in literacy. A tribute to Isabelle Liberman (pp. 5-27). Hillsdale, NJ: Erlbaum

Nunes, T., Bryant, P., y Olson, J.M. (2003): Learning morphological and phonological spelling rules: an intervention study. Reading and Writing: An Interdisciplinary Journal, 63, 141-170.

Nunes, T., y Bryant, P. (2004). Morphological awareness improves spelling and vocabulary. Literacy Today, 11, 1-19.

Rodrigo, M., Jiménez, J.E., García, E., Díaz, A., Ortiz, M.R., Guzmán, R., Hernández-Valle, I., Estévez, A., y Hernández, S. (2004). Valoración del procesamiento ortográfico en niños españoles con dislexia: El papel de las unidades léxicas y subléxicas. Revista Electrónica de Investigación Psicoeducativa y Psicopedagógica, 2(2), 105-126.

Seymour, P.H.K. (1997). Foundations of orthographic development. En Perfetti, C. A.; Rieben, L. y Fayol (Eds). Learning to spell: Research, theory, and practice across languages (pp. 319-337). Mahwah, NJ: Erlbaum.

Recibido: 27 de mayo de 2018 Recepción modificaciones: 9 de septiembre de 2018 Aceptado: 10 de septiembre de 2018 